FAITS ET CHIFFRES

\title{
Carrières d'agriculteurs et transformation des structures des exploitations agricoles
}

Farmers individual careers and transformation of farm structures

\author{
Gilles Allaire et Élise Maigné
}

\section{(2) OpenEdition \\ Journals}

\section{Édition électronique}

URL : http://journals.openedition.org/economierurale/5161

DOI : 10.4000/economierurale.5161

ISSN : 2105-2581

Éditeur

Société Française d'Économie Rurale (SFER)

Édition imprimée

Date de publication : 17 février 2017

Pagination : 123-144

ISSN : 0013-0559

Référence électronique

Gilles Allaire et Élise Maigné, "Carrières d'agriculteurs et transformation des structures des exploitations agricoles », Économie rurale [En ligne], 357-358 | janvier-mars 2017, mis en ligne le 17 février 2019, consulté le 19 avril 2019. URL : http://journals.openedition.org/economierurale/5161 ; DOI : 10.4000/economierurale.5161 


\title{
FAITS ET CHIFFRES
}

\section{Carrières d'agriculteurs et transformation des structures des exploitations agricoles}

\author{
Gilles ALLAIRE • Élise MAIGNÉ \\ INRA, US-ODR, Castanet-Tolosan \\ gilles.allaire@inra.fr
}

\begin{abstract}
S'agissant des exploitations familiales, les transformations des structures de production sont liées à la dynamique de carrière des exploitants : installation, intégration de successeurs ou de collaborateurs familiaux, préparation de la retraite, voire renoncement ou réorientation en cours de carrière. L'étude présentée ici porte sur l'analyse des carrières des exploitants cotisant à la Mutualité sociale agricole. Elle repose sur la constitution d'une base de données individuelles longitudinales permettant d'analyser les transitions d'une année sur l'autre au cours de la période 2003-2013, s'agissant du régime d'activité (pluriactif ou non) et du type d'exploitation (structure du collectif de travail) sur laquelle exerce un exploitant. La répartition des types de structures (collectifs de travail) distingués est plutôt stable sur la période : les exploitations unipersonnelles dominent largement aux deux dates. II ressort de l'analyse des carrières que les dynamiques concernent deux ensembles qui se distinguent, une grande majorité d'exploitations où un exploitant est seul présent (avec ou sans support familial ou salarié, $2 / 3$ des exploitants) et une minorité d'exploitations familiales complexes.
\end{abstract}

\section{MOTS-CLÉS : carrières agricoles, trajectoires professionnelles, structures agricoles}

\section{Farmers individual careers and transformation of farm structures}

For family farms the transformations of structures of production are related to the dynamics of career of operators (considering non-wage farmers) installation, integration of successors or family collaborators, retirement planning, or even renunciation or reorientation during their career. The study presented here is on the analysis of the careers of the operators contributing to the social agricultural mutual insurance system and is based on the constitution of a longitudinal individual data base to analyze transitions from one year to another during the period 2003-2013 related to two parameters: the regime of activity (part-time or not) and the type of operation (structure of the working collective) in which is present an operator. The distribution of operators the types of structures distinguished is rather stable over the period: one-person operations dominate on the two dates. It is clear from the analysis of the careers that the dynamics relates to two sets that stand out, a majority of operations where an operator is only present (with or without family support or employees, 2/3 of the operators) and a minority of complex family farms. (JEL : C81, J11, Q12).

KEYWORDS: farmers' professional career, entry in agriculture, retirement

C t article s'intéresse aux structures productives de l'agriculture familiale en considérant les carrières individuelles des agriculteurs du point de vue du régime d'activité et des transformations structurelles des collectifs de travail. Il y a un lien depuis longtemps mis en évidence par l'économie rurale entre le cycle familial et la dynamique des structures de production avec des phases de croissance et de régression ; ainsi l'entrée dans le collectif de travail familial d'un enfant est souvent concomitante d'un accroissement de superficie et, en fin de cycle, en l'absence de successeur nous observons fréquemment une diminution de l'activité. L'originalité de cet article est d'appréhender les carrières individuelles des agriculteurs, en observant annuellement les changements qui peuvent intervenir soit dans le régime 
d'activité de l'individu (mono- ou pluriactivité), soit dans le type de structure d'exploitation (définie par son collectif de travail) sur laquelle il exerce son activité.

La population active agricole familiale est composée d'exploitants et d'aides familiaux au sens large (enfants, conjoints, parents). L'identification des différentes catégories d'actifs ressort des évolutions de la protection sociale agricole, qui a été mise en place dans les années 1950 , d'abord essentiellement pour les «chefs d'exploitation » et la population masculine. Plus tard, les statuts d'aide familial et de conjoint actif sur l'exploitation ont été reconnus et précisés dans le but d'assurer une couverture sociale individuelle. Mais l'emploi des jeunes en tant qu'aide familial régresse fortement depuis au moins trois décennies, ceux qui deviennent agriculteurs s'installant en tant qu'exploitant après les études ou une activité hors de l'exploitation familiale ou commence à travailler comme salarié sur celle-ci ; par ailleurs, des installations de plus en plus nombreuses s'effectuent hors du cadre familial. Les femmes agricultrices optent de plus en plus pour le statut d'exploitante plutôt que «conjointe participant aux travaux ». La carrière d'agriculteur se déroule donc de plus en plus essentiellement avec le statut d'exploitant (cotisant à l'Assurance maladie des exploitants agricoles ou AMEXA). La présente étude est consacrée aux carrières des exploitants, qui aujourd'hui représentent plus de $90 \%$ de la population active agricole non salariée connue par la protection sociale agricole, carrières que nous étudions à partir de cette source (MSA), au long de la décennie 2003-2013. L'évolution du nombre d'exploitants résulte des départs en retraite, des mouvements d'entrée et de sortie en cours d'activité s'effectuent à tout âge et du taux de renouvellement. Elle dépend donc, dans son rythme, de la démographie, du contexte socioéconomique et des politiques structurelles (abaissement de l'âge de la retraite dans les années 1990, puis allongement de la durée de cotisation au cours de la période étudiée, etc.).

Le changement de régime d'activité, agriculteur à titre exclusif, principal ou secondaire, est un élément marquant des carrières. Peu d'entrants pluriactifs conservent ce statut tout au long de leur carrière, soit ils quittent la carrière agricole, soit leur activité non agricole, et, en sens inverse, une activité complémentaire peut intervenir à différents moments d'une carrière. L'étude des transitions sur une cohorte permet de mieux connaître ces dynamiques. Une autre variable clé des carrières agricoles est la modification de la composition du collectif de travail. Différentes évolutions sont possibles selon que l'installation s'effectue de façon indépendante ou dans le cadre d'un collectif familial et selon la présence ou non d'un successeur familial. Ces changements concernent la structure de l'exploitation. Pour les étudier, nous avons défini des types d'exploitation selon la composition du collectif de travail.

Dans l'analyse des carrières d'agriculteurs, nous nous intéresserons particulièrement aux phases de début et de fin, en observant sur 10 ans la cohorte des entrants dans l'activité d'exploitant agricole en 2003 et les 10 dernières années de carrière pour les sortants en 2012. L'objectif est de mieux connaître les trajectoires lors de ces phases qui sont non seulement importantes du point de vue de la gestion des carrières par les individus mais aussi parce qu'elles sont accompagnées par la politique agricole.

Nous présenterons d'abord les données utilisées et les caractéristiques de l'emploi agricole à partir de la source mobilisée (données de la MSA, mises à disposition de L'INRA via l'Observatoire du développement rural [ODR]). Elles permettent d'observer les carrières individuelles des exploitants, non-salariés, cotisant à l'Assurance maladie des chefs 
d'exploitation agricole (AMEXA) sur une période de 10 ans, du $1^{\text {er }}$ janvier 2003 au

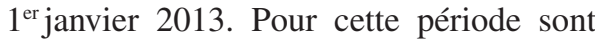
étudiées toutes les transitions d'une année sur l'autre, pour lesquelles nous pouvons observer ou non un changement de régime d'activité ou de structure d'exploitation. Pour caractériser ces transitions sont considérés les attributs des exploitations sur lesquelles sont présents les exploitants chaque $1^{\text {er }}$ janvier, qu'il s'agisse de la même exploitation que l'année précédente ou non, en termes de statut juridique ou de siège. Nous analyserons d'abord les transitions pour toute la population présente au cours de la période puis pour les cohortes des entrants et des sortants. Les transitions sont étudiées selon le genre (Hommes, Femmes) et l'âge.

\section{Données sur l'emploi agricole non salarié et méthode d'analyse des carrières}

La caisse centrale de la Mutualité sociale agricole (ci-après MSA) fournit des statistiques de l'emploi salarié et non-salarié agricole au $1^{\text {er }}$ janvier de chaque année à partir des fichiers de cotisants aux différents régimes de protection sociale agricole $^{1}$. Le champ des activités «agricoles » de la MSA est différent de celui des recensements et enquêtes réalisés par l'Insee et le Service de la statistique et de la prospective (SSP) du ministère de l'Agriculture. Il inclut la filière bois (sylviculture, exploitation de bois, scieries), une partie des métiers de la mer ou du monde hippique, les artisans ruraux, les entreprises de travaux agricoles, de jardins et paysagistes. Le fichier annuel des cotisants

1. Il est possible de consulter des tableaux de bord de l'emploi salarié et non salarié sur le site de l'Observatoire du développement rural (ODR). Les bases de données individuelles correspondantes (gérées par Élise Maigné) ont été utilisées pour cette étude. non-salariés (COTNS) rassemble tous les chefs d'exploitation ou d'entreprise en activité, qui cotisent à au moins une des 4 branches : maladie (AMEXA), prestations familiales, vieillesse ou accidents du travail (ATEXA). Toutefois, seulement les agriculteurs peuvent cotiser à l'Assurance maladie des chefs d'exploitation agricole (AMEXA), sous réserve d'un volume d'activité agricole suffisant. Ce critère nous a permis d'exclure de l'analyse les chefs d'entreprise non agricoles au sens de la statistique agricole (Agreste). Dans ce fichier des cotisants, les individus sont rattachés à une entreprise identifiée par un numéro Siret, ce qui permet de dénombrer les exploitants d'une même exploitation à une date donnée et d'y rattacher une quantité de travail salarié en faisant le lien avec la base annuelle SISAL des contrats de salariés agricoles. Par définition, COTNS ignore les exploitations agricoles en société gérées par des salariés ou des établissements publics, donc une partie des structures de production les plus importantes (les exploitations agricoles présentes dans la base SISAL et absentes de COTNS représentent un peu plus de $20 \%$ de l'ensemble des exploitations agricoles (compte non tenu des entreprises MSA non agricoles au sens strict) présentes dans les 2 bases, en 2012). La présente étude se rapporte à l'agriculture professionnelle familiale, c'est-à-dire aux exploitations agricoles dirigées par un ou plusieurs nonsalariés.

\section{Définition du champ de l'étude}

À partir des fichiers des cotisants nonsalariés (COTNS) et de la base SISAL a été construite une base longitudinale permettant un suivi des individus pour la période 2003-2013, donnant chaque année, pour chaque chef d'exploitation, des caractéristiques individuelles (statut MSA, statut matrimonial, surface détenue, etc.) ainsi que des caractéristiques de l'exploitation sur laquelle il se situe (nombre 
Tableau 1. Emploi agricole non-salarié en 2013 (champ de l'étude)

\begin{tabular}{lrrr}
\hline \multirow{2}{*}{ Statut MSA cotisants en 2013 } & \multicolumn{3}{c}{ Individus dans le champ de l'étude } \\
\cline { 2 - 4 } & $\begin{array}{r}\text { TOTAL } \\
\text { AMEXA }\end{array}$ & $\begin{array}{r}\text { Dont non } \\
\text { AMnt cotisants soli- } \\
\text { daires (<1/2 SMI) }\end{array}$ \\
\hline Chefs d'exploitation ou d'entreprise agricole à titre exclusif & 412363 & 11782 & 11341 \\
Chefs d'exploitation ou d'entreprise agricole à titre principal & 31608 & 601 & 546 \\
Chefs d'exploitation ou d'entreprise agricole à titre secondaire & 46614 & 11182 & 8592 \\
Ensemble des chefs d'exploitation ou d'entreprise agricole & 490585 & 23565 & 20479 \\
Nombre d'exploitations correspondantes & 396776 & & \\
*Conjoints collaborateurs ou participants aux travaux & 38805 & & \\
*Aides familiaux & 4584 & & \\
\hline
\end{tabular}

Note : * Pour ces catégories il n'y a pas d'identifiant individuel dans COTNS.

Source : MSA, COTNS, ODR.

d'exploitants associés, travail salarié, surface). Pour la présente étude, la population de la base longitudinale 2003-2013 a été restreinte aux chefs d'exploitation cotisant à l'AMEXA au moins une année durant la période. L'appellation chef d'exploitation ou d'entreprise, utilisée par la MSA, correspond aux notions de chef d'exploitation et de co-exploitant dans les statistiques agricoles; aussi parlerons-nous dans la suite indifféremment de chef d'exploitation ou d'exploitant.

La définition du champ d'étude élimine ceux des « cotisants solidaires » (non cotisants à l'AMEXA) qui conservent ce statut pour toute la durée de leur présence dans COTNS durant la période étudiée $^{2}$. Par contre, elle inclut ceux qui sont devenus cotisants AMEXA durant

2. Seule une personne physique peut être « cotisant de solidarité », sous réserve d'un acte d'exploitation procurant des revenus et dans la mesure où cette activité est insuffisante pour accéder au statut d'exploitant agricole. Sauf les cas d'installation progressive avec accès au statut d'exploitant (AMEXA), il ne s'agit pas d'une véritable activité professionnelle agricole. Un tiers environ sont des retraités agricoles détenant une exploitation de subsistance supérieure à 2 ha, les individus qui étaient dans cette situation en 2003 ne font pas partie de la cohorte qui, par contre, comprend ceux qui étaient exploitants et sont devenus retraités cotisant solidaires. la période (7 042 personnes sur 559464 présentes en 2003 , soit $1,3 \%$ ) ou l'étaient avant de devenir «solidaires » (20 943 sur 490585 exploitants présents en 2013, soit $4,3 \%$ ) afin d'inclure installations et retraites progressives. Ainsi, le nombre de cotisants solidaires dans le champ de l'étude s'accroît au long de la période. Les autres personnes qui ne cotisent pas à l'AMEXA durant toute la période d'étude sont des personnes qui viennent ou qui sont allées vers une activité non agricole au sens retenu (exploitation de bois, centre hippique...); leur nombre est très faible ( 3086 personnes autres que cotisants solidaires, $0,6 \%$ en 2013 , $c f$. $t a$ bleau 1). La population suivie passe de 559464 exploitant(e)s en 2003 à 490585 en 2013, avec un effectif moyen annuel de 525763 individus (cf. tableau 5).

Les exploitants cotisant à l'AMEXA et les cotisants solidaires sont distingués selon qu'ils cotisent à un ou plusieurs régimes, le régime principal étant alors défini par l'activité qui produit le plus de revenu ou occupe le plus du temps de travail. Trois régimes d'activité sont ainsi distingués : non-salariés agricoles à titre «exclusif », « principal » et « secondaire » (tableau 1).

Le taux de pluriactivité de la population étudiée (exploitants à titre principal et secondaire) de 2003 à 2013 passe de 14 à 
$16 \%$. Cette augmentation de la pluriactivité est confirmée par les résultats des recensements agricoles qui montrent également une augmentation sur la décennie 2000 après une stabilité sur la décennie 1900 (tableau 2) (l'écart avec nos données est lié à la définition du champ d'étude). On observe sur la période une augmentation du nombre d'exploitants à titre secondaire dans la cohorte (40 430 [7,2\%] en 2003 et 46 614, [9 $5 \%$ ], en 2013, soit une augmentation de $+15 \%$ ), avec un maximum de 47556 exploitants en $2011^{3}$, tandis que le nombre total d'individus (tous régimes) chute de $12 \%$. Cette augmentation se traduit par une augmentation de la part des femmes dans cette catégorie qui passe de $23,4 \%$ en 2003 à 27,4 \% en 2013. La part des femmes parmi les exploitants à titre principal augmente également, de $24,5 \%$ à $27,1 \%$. Ceci reflète une évolution réglementaire et sans doute sociale du statut des agricultrices.

Tableau 2. Pluriactivité des chefs d'exploitation et co-exploitants selon les recensements agricoles

\begin{tabular}{lccc}
\hline Recensements agricoles & 1988 & 2000 & 2010 \\
\hline Total pluriactifs & $19,3 \%$ & $19,2 \%$ & $21,5 \%$ \\
\hline
\end{tabular}

Source : Agreste, d'après Graph'agri 2012.
Pour situer la population active non salariée dans l'ensemble de la population active agricole, le tableau 3 fournit le décompte des salariés pour l'année 2012. En 2012, 608004 salariés fournissent (a) 155614 Équivalents temps plein (ETP) à l'activité agricole des exploitations familiales. Si celles-ci comptent pour $78 \%$ des établissements avec salariés agricoles, elles n'utilisent que $56 \%$ de cette force de travail (déclarée). Elles réunissent par ailleurs (b) 533974 actifs (tableau 1), dont la durée d'activité annuelle n'est pas connue, mais en moyenne inférieure à 1 ETP; la part du salariat dans les exploitations agricoles familiales est donc au minimum de $23 \%[\mathrm{a} /(\mathrm{a}+\mathrm{b})]$.

\section{Mouvements d'entrée et de sortie de l'emploi agricole des exploitants non salariés}

Chaque année, les entrées dans la cohorte (l'exploitant n'était pas présent l'année $\mathrm{N}-1$ dans la base et apparaît en $\mathrm{N}$ ) sont des installations agricoles et les sorties (l'exploitant était présent l'année $\mathrm{N}$ et n'est plus présent en $\mathrm{N}+1$ ) sont des sorties définitives de l'activité d'exploitant agricole (le nombre de départs de la cohorte suivis d'une nouvelle entrée est négligeable,

Tableau 3. Emploi agricole salarié en 2012

\begin{tabular}{|c|c|c|c|c|c|}
\hline & \multirow{2}{*}{$\begin{array}{l}\text { Nombre } \\
\text { d'établis- } \\
\text { sements }\end{array}$} & \multicolumn{3}{|c|}{ Nombre de salariés } & \multirow{2}{*}{$\begin{array}{l}\text { Équivalents } \\
\text { temps plein }\end{array}$} \\
\hline & & Total & Hommes & Femmes & \\
\hline $\begin{array}{l}\text { Établissements agricoles figurant } \\
\text { dans COTNS au 1/1/2013 (dirigés } \\
\text { par des non-salariés) }\end{array}$ & $112327(78 \%)$ & 608004 & 375238 & 232766 & $155614(56 \%)$ \\
\hline $\begin{array}{l}\text { Établissements ayant une activité } \\
\text { agricole non présents dans COTNS } \\
\text { au 1/1/2013 (dirigés par des salariés) }\end{array}$ & $32525(22 \%)$ & 349192 & 211989 & 137203 & $122558(44 \%)$ \\
\hline
\end{tabular}

Source : MSA, SISAL, COTNS, tableaux de bord de l'emploi agricole, ODR (champ agricole défini par l'ATEXA).

3. Ceci est en partie un artefact dû à notre définition de la cohorte étudiée qui s'enrichit en cotisants solidaires, leur poids passant de $1,3 \%$ en 2003 à $4,3 \%$ en 2013 et ceux-ci représentant $15,6 \%$ des secondaires en 2003 et $18,8 \%$ en 2013. 


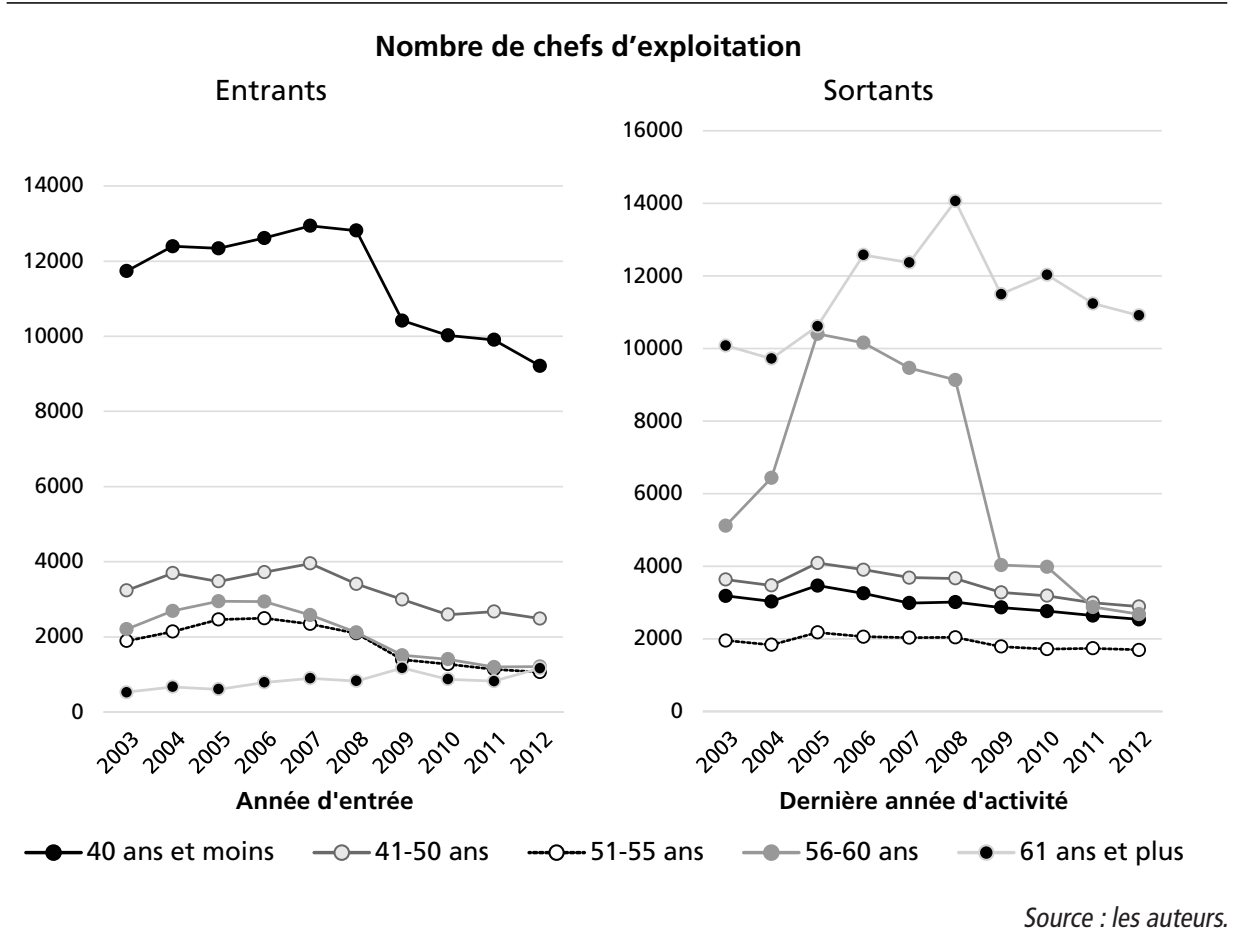

791 cas pour la décennie étudiée). Pour la période étudiée, en moyenne, le nombre d'entrées par an est de 19412 exploitants (4,5\% de l'effectif moyen) et celui des sorties de $26302(6,1 \%)$.

Si une majorité des entrants ont 40 ans et moins et si la grande majorité des sortants accède à la retraite, contrairement à la représentation courante d'une stabilité de la profession d'agriculteur, des mouvements d'entrée et de sortie en cours d'activité s'effectuent à tout âge (graphique 1). Les taux de sortie varient, comme nous le verrons, selon le régime d'activité, la taille et la structure d'exploitation. Les sortants en fin de carrière (61 ans et plus ainsi que la majeure partie des 56-60 ans) augmentent de 2005 à 2008 du fait des classes «pleines » de l'après Seconde Guerre et du bénéfice de la réforme des retraites concernant les carrières longues. Par contre le nombre des sortants diminue ensuite, entraînant une chute des entrées.

Dès 2002, une étude du CNASEA (Bernardi et Lefebvre, 2002) utilisant également le fichier COTNS de la MSA soulignait l'importance des départs « précoces » (avant 55 ans) de l'agriculture : ainsi entre 1997 et 1999, au niveau national, 1/3 des sortants sont des exploitants de moins de $55 \mathrm{ans}^{4}$. Au début de notre période d'étude (2003) les sorties précoces représentent $37 \%$ des sortants, cette proportion baisse (31\% en 2005) pour remonter en fin de période (35\% en 2012).

4. C'est également une période où le nombre de sorties a fortement diminué après la forte hausse des départs déclenchée par le premier dispositif de préretraite (de 1993 à 1996) (Allaire et Daucé, 1996) et du fait des classes «creuses » nées avant la Seconde Guerre. 


\section{3. Étude des transitions annuelles dans les carrières d'agriculteurs}

Nous décrivons les carrières d'agriculteurs comme une série de transitions annuelles, que l'on peut observer à partir de la base longitudinale que nous avons constituée.

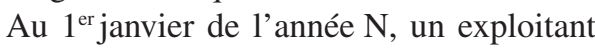
est caractérisé par des paramètres d'état, dont la valeur change ou non l'année suivante $(\mathrm{N}+1)$; lors de la transition annuelle, il y a ou non un changement d'état significatif dans la carrière. Nous considérons ici seulement deux paramètres : le régime d'activité (section 3) ; le type de structure d'exploitation (défini selon la composition du collectif de travail, ci-dessous) (section 4), en séparant les trajectoires en début de carrière agricole (cohorte des entrées en 2003) (section 5) et en fin de carrière (cohorte des sorties 2012) (section 6).

Nous analysons les changements d'état en constituant des matrices de transition qui indiquent la fréquence des changements d'état pris deux à deux en considérant ensemble toutes les transitions annuelles observées sur la période de 10 ans (entre 2003 et $2004, \ldots, \mathrm{N}$ et $\mathrm{N}+1, \ldots, 2012$ et 2013). Les tableaux ci-dessous présentent des matrices de changements entre une année $\mathrm{N}$ et la suivante $(\mathrm{N}+1)$, en utilisant la moyenne des 10 matrices établies sur la période, sauf dans le cas des entrées pour lequel on comparera avec la moyenne de la première période de 3 ans. Un paramètre d'état est stable lorsque l'exploitant est présent à deux dates successives et que l'état est le même. Les entrées et les sorties sont des transitions particulières et figurent dans les matrices de transition.

\section{Types d'exploitation}

Avant de passer à l'analyse des matrices de transition, nous définissons les types d'exploitation pris en compte pour caractériser les changements de structure selon la taille et la composition du collectif de travail : nombre d'exploitants $(1,2$, plus de 2) et présence d'un «support familial » ou d'un « support salarié » (l'apport salarié étant mesuré par le lien avec la base SISAL ${ }^{5}$ ). Nous définissons comme support familial la présence de conjoints «participant aux travaux » ou «collaborateurs» ou d'«aides familiaux» (personnes rattachées à un exploitant pour la cotisation retraite). Depuis 2005, le statut de conjoint participant aux travaux n'existe plus, ce qui conduit à des installations de conjointes comme exploitantes plus fréquentes. L'aide familial est un membre de la famille en dehors du conjoint qui participe à la mise en valeur de l'exploitation: ascendant ou, à partir de 16 ans, descendant, frère, sœur ou allié au même degré du chef d'exploitation ou de son conjoint. Depuis 2006, le statut d'aide familial est limité à 5 ans. L'effectif des conjoints et aides familiaux se réduit en conséquence bien plus fortement que le nombre d'exploitants : $-9 \%$ sur la période pour les exploitants, $-61 \%$ pour les conjoints et $-56 \%$ pour les aides familiaux. Il est important de noter que l'apport de travail familial ne se limite pas aux cas que l'on peut repérer dans le fichier COTNS à partir des cotisations additionnelles payées par un exploitant pour son conjoint ou des aides familiaux ; en effet, des conjoints salariés, des enfants étudiants ou des parents retraités apportent généralement des «coups de main » plus ou moins cruciaux lorsqu'ils sont présents.

La typologie présentée dans le tableau 4 est ordonnée selon la complexité du collectif de travail de l'exploitation (hors travail informel ou illégal évidemment); les changements qui s'opèrent $\mathrm{du}$ haut vers le bas du tableau correspondent à une phase de croissance ou de

5. Pour une année $\mathrm{N}$, l'affectation d'un support salarié aux exploitations présentes dans COTNS (au $1^{\text {er }}$ janvier) est faite selon l'observation des contrats de travail en N-1 (SISAL). De ce fait une petite partie des entrées dans le cas 1_0 apparâ̂tront 1_S l'année N+1. 
Tableau 4. Définition des types d'exploitation selon la structure du collectif de travail

\begin{tabular}{|c|c|c|c|}
\hline $\begin{array}{l}\text { Type de } \\
\text { structure }\end{array}$ & $\begin{array}{c}\text { Superficie } \\
\text { moyenne } \\
2013 \\
\end{array}$ & Définition & Type simplifié \\
\hline 10 & 41 & unipersonnelle sans support & \multirow{4}{*}{ Exploitations unipersonnelles } \\
\hline 1_F & 58 & unipersonnelle avec support familial & \\
\hline 1_S & 50 & unipersonnelle avec support salarié & \\
\hline 1_M & 57 & unipersonnelle avec support mixte (familial et salarié) & \\
\hline 2_0_A & 104 & $\begin{array}{l}\text { bipersonnelle sans support, les deux chefs sont de la } \\
\text { même génération }\end{array}$ & \multirow{5}{*}{ Exploitations bipersonnelles } \\
\hline $2 \_0 \_B$ & 115 & $\begin{array}{l}\text { bipersonnelle sans support, les deux chefs ne sont pas } \\
\text { de la même génération }\end{array}$ & \\
\hline 2_F & 126 & bipersonnelle avec support familial & \\
\hline $2 S$ & 107 & bipersonnelle avec support salarié & \\
\hline 2_M & 138 & bipersonnelle avec support mixte (familial et salarié) & \\
\hline 3 & 169 & plus de deux chefs d'exploitation & Exploitations pluripersonnelles \\
\hline
\end{tabular}

Source : les auteurs.

complexification du collectif de travail et, en sens inverse, à une phase de déclin ou de simplification.

Au total, $84 \%$ en 2003 et $81 \%$ en 2013 des exploitations de notre champ d'étude (dirigées par un ou plusieurs non-salariés) sont unipersonnelles ; elles sont mises en valeur par $69 \%$ en 2003 et $65 \%$ en 2013 des exploitants (voir les chiffres dans les 2 premières colonnes du tableau 5). Les cas d'exploitant seul avec un complément de main-d'œuvre (1_F, 1_S, 1_M) représentent environ un tiers des exploitations (35\% en 2003 et $30 \%$ en 2013) et un peu moins d'exploitants (29\% et $24 \%$ respectivement). Entre ces deux dates, la superficie moyenne des exploitations (60 ha en 2013) augmente de $10 \%$; celle des exploitations unipersonnelles augmentant moins que la moyenne. Les types avec support familial ont fortement régressé du fait des changements réglementaires.

L'importance des exploitations unipersonnelles ne correspond sans doute pas tout à fait aux réalités économiques. Dans certains cas (en particulier dans les régions de faire-valoir direct comme le Sud-Ouest), il est vraisemblable que des exploitations de ce type appartiennent à des membres d'une même famille (père et fils) et sont complémentaires ou constituent de fait une même entreprise. Dans d'autres cas, plutôt que louer leurs terres à un fermier, certains propriétaires préfèrent bénéficier du statut d'agriculteur et faire travailler les terres « à l'entreprise » par un agriculteur voisin. Il y a là un biais propre aux données MSA par rapport aux réalités économiques. L'unité statistique est l'exploitant et non l'exploitation. Plusieurs exploitants ne peuvent être rattachés à une même exploitation que pour autant que celle-ci ait un statut juridique.

Les graphiques du nombre d'exploitations par type tout au long de la période montrent l'augmentation à partir de 2008 des exploitations avec support salarié au détriment du support familial (en partie du fait de la modification du régime des conjoints) tandis que le nombre de cas «mixtes» (support salarial et familial : 1_M et 2_M), au demeurant faible, ne diminue que lentement (graphique 2). Nous reviendrons sur cette question avec l'étude des transitions. 
Graphique 2. Nombre d'exploitations par type et par année

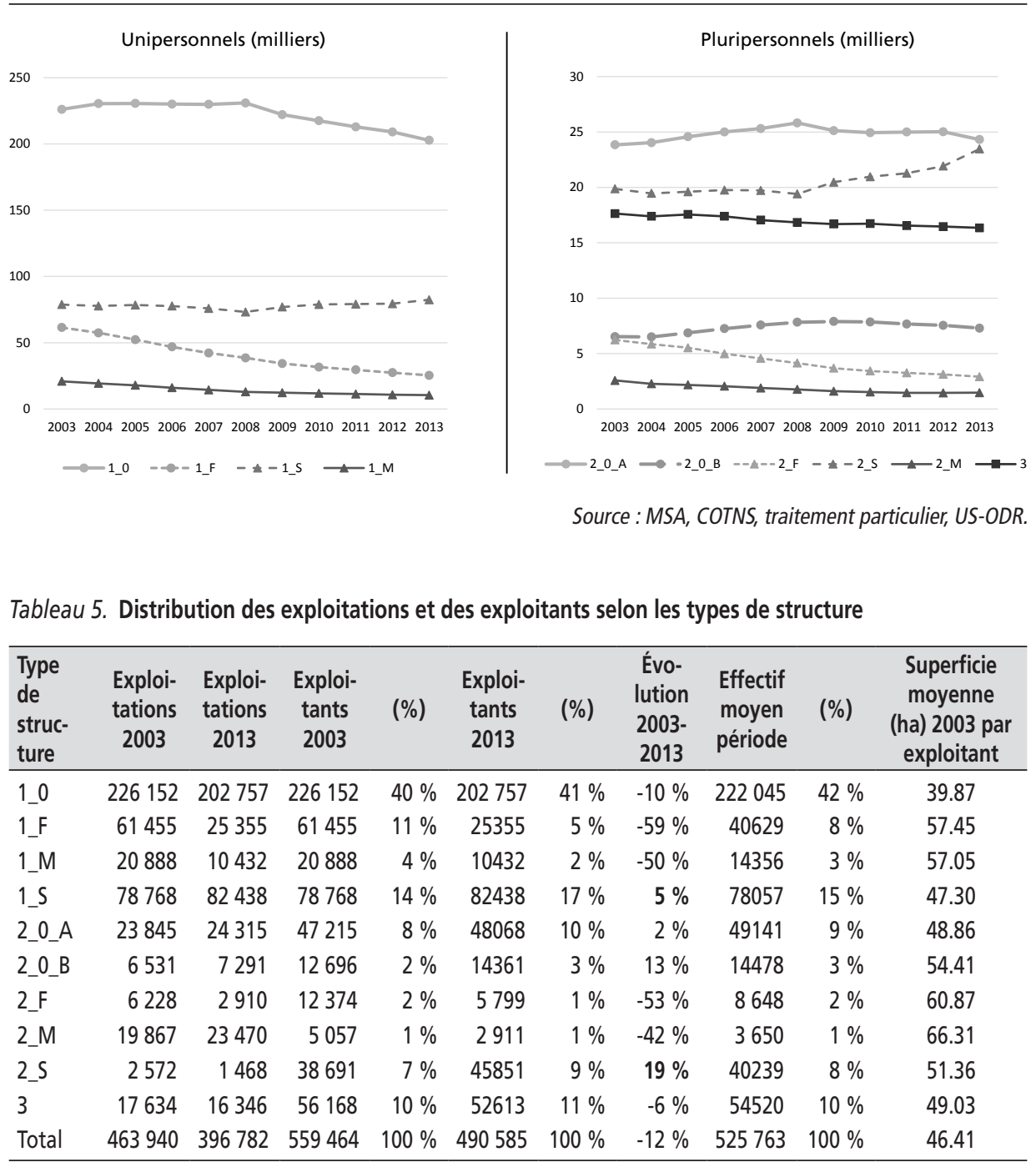

Source : MSA, COTNS, traitement particulier, US-ODR.

\section{Distribution des exploitants selon} les types de structure en 2003 et en 2013

Le nombre d'exploitants diminue moins que le nombre d'exploitations pour le champ et la période d'étude : $-12 \%$ ( $t a$ bleau 5) au lieu de $-14,5 \%$, du fait du maintien des exploitations bipersonnelles (59043 en 2003 et 59454 en 2013, pour les 5 types 2) et de la diminution légère des pluripersonnelles $(-7 \%)$ en comparaison des unipersonnelles $(-17 \%$; toutefois, on observe, pour ce type, une augmentation des exploitations avec salariés).

\section{Transitions entre régimes d'activité}

L'étude des transitions annuelles ( $t a$ bleau 6) montre que les changements de régimes sont nettement plus fréquents vers le régime d'exploitant à titre exclusif (qui 
Tableau 6. Matrice de transition entre régimes d'activité, moyennes sur 2003-2013

\begin{tabular}{l|c|c|c|c|c|c|c}
\hline $\mathbf{N}$ & Exclusif & Principal & Secondaire & Sortie & Effectif & $\begin{array}{c}\text { Nombre de } \\
\text { sorties }\end{array}$ & $\begin{array}{c}\text { Sorties selon } \\
\text { régimes }\end{array}$ \\
\hline Exclusif & 94,1 & 0,7 & 0,3 & 4,8 & 450788 & 21773 & $82,9 \%$ \\
Principal & 9,4 & 81,9 & 3,8 & 5 & 33784 & 1679 & $6,4 \%$ \\
Secondaire & 5,1 & 1,8 & 86,8 & 6,3 & 44710 & 2817 & $10,7 \%$ \\
Entrée selon régimes & 73,4 & 6,8 & 19,9 & 0 & 19412 & 0 & 0 \\
\hline
\end{tabular}

Note : en $\%$ des lignes.

Source : MSA, COTNS, traitement particulier, US-ODR.

Tableau 7. Régime maladie des entrants en 2003 et présence en 2013

\begin{tabular}{l|rr|rr|c}
\hline $\begin{array}{l}\text { Statut } \\
\text { des entrants }\end{array}$ & \multicolumn{2}{|c|}{2003} & \multicolumn{2}{c|}{ Toujours actifs en 2013 } & $\begin{array}{c}\text { Variation 2003 2013 } \\
\text { (taux de sortie }\end{array}$ \\
\hline Exclusif & 14337 & $71,36 \%$ & 10305 & $79,98 \%$ & $-28 \%$ \\
Principal & 1302 & $6,48 \%$ & 914 & $7,09 \%$ & $-30 \%$ \\
Secondaire & 4451 & $22,16 \%$ & 1665 & $12,92 \%$ & $-63 \%$ \\
Total & 20090 & $100,00 \%$ & 12884 & $100,00 \%$ & $-36 \%$ \\
\hline
\end{tabular}

Source : MSA, COTNS, traitement particulier, US-ODR.

est le régime le plus stable) que l'inverse ; ceci est également vrai en nombre absolu: en moyenne, $1 \%$ des exclusifs soit 4508 individus deviennent pluriactifs tandis que 5456 pluriactifs (cumul des lignes secondaire et principal du tableau 6) deviennent exploitants à titre exclusif.

Lecture du tableau : 94,1\% des exploitants à titre exclusif conservent ce statut d'une année sur l'autre, tandis que 4,8\% sortent de l'agriculture (en moyenne chaque année). Des exploitants à titre principal abandonnent leur activité non agricole et accèdent au régime à titre exclusif $(9,4 \%)$, tandis que $5 \%$ sortent, etc. $73,4 \%$ des entrées sont des exploitants à titre exclusif, la dernière colonne indique qu'il s'agit de 82, $9 \%$ des sortants.

Il y a deux aspects complémentaires de l'évolution de la cohorte. D'un côté, chaque année, les exploitants à titre secondaire qui abandonnent la carrière agricole $(6,3 \%)$ sont presque aussi nombreux que ceux qui transitent vers le régime principal $(1,8 \%)$ ou exclusif $(5,1 \%)$. La proportion du nombre d'exclusifs se renforce donc au sein de la cohorte au fil des années. Alors que le poids des secondaires est dans les entrées de $19,9 \%{ }^{6}$, il n'est plus que de $10,7 \%$ dans les sorties (en moyenne pour les années étudiées). Cette évolution se retrouve dans la dynamique de la cohorte des entrées en 2003, les secondaires qui étaient au départ de 22,2\% ne sont plus que $12,9 \%$ parmi ceux qui sont toujours actifs en 2013 (tableau 7); près des 2/3 ont quitté la carrière agricole.

Seulement $24 \%$ des exploitants entrés en 2003 connaissent un ou plusieurs changements de régime durant la décennie et pour l'essentiel (17\%) nous n'observons qu'un seul changement. Les transitions pour cette cohorte ont été étudiées en considérant soit la moyenne des transitions pour toute la période, soit seulement pour les 3 années suivant l'entrée (matrices 2003-2004, 2004-2005 et 2005-2006)

6. Les entrées comme secondaires représentent $25 \%$ des entrées pour la classe d'âge 41-50 ans et seulement $18 \%$ des entrées des 40 ans et moins. 
Tableau 8. Matrice de transition entre régimes de la cohorte des entrants 2003

\begin{tabular}{l|c|c|c|c|c|c}
\hline Transitions & N & Exclusif & Principal & Secondaire & Taux Sortie & Effectif \\
\hline Toutes & \multirow{2}{*}{ Exclusif } & 93,6 & 1,1 & 0,5 & 4,9 & 12640 \\
3 premières & & 92,8 & 1,4 & 0,6 & 5,4 & 14352 \\
\hline Toutes & \multirow{2}{*}{ Principal } & 12,1 & $\mathbf{7 9 , 2}$ & 3,6 & 5,1 & 1133 \\
3 premières & & 16,8 & $\mathbf{7 5 , 2}$ & 3,0 & 4,9 & 1271 \\
\hline Toutes & \multirow{2}{*}{ Secondaire } & 6,9 & 2,1 & $\mathbf{8 4 , 8}$ & 6,2 & 2450 \\
3 premières & & $\mathbf{1 2 , 6}$ & $\mathbf{2 , 6}$ & $\mathbf{7 6 , 9}$ & $\mathbf{7 , 9}$ & 3423 \\
\hline
\end{tabular}

Note: en $\%$ des lignes.

(tableau 8). L'instabilité est la plus grande lors des 3 premières années de la carrière, mais les taux de sortie peu différents, sauf pour les secondaires. Les changements vers le statut d'exploitant à titre principal s'effectuent en début de carrière, 16,8\% en moyenne chaque année lors des 3 premières années de la carrière pour ceux dont l'activité agricole est principale et $12,6 \%$ pour les secondaires (alors que les autres changements ne sont en moyenne pas plus fréquents lors de ces 3 ans que sur toute la période).

\section{Étude des transitions entre types de structure d'exploitation}

Nous décrivons d'abord la matrice des transitions (tableau 9) qui indique les fréquences annuelles moyennes (sur la période) des changements d'état (pour un exploitant, le passage d'un type de structure à un autre ou la sortie). Puis nous étudierons les transitions sans changement d'état, c'est-à-dire les cas avec stabilité de la structure d'exploitation (86\% en moyenne), puis les changements dans le sens d'un accroissement ou d'une régression de la structure. Les attributs analysés sont des moyennes pour toutes les transitions sur la durée de la période (ou la médiane pour l'âge).

Les cases à droite de la diagonale du tableau (cases encadrées) correspondent à un développement, les fréquences de changement significatives correspondent soit à un développement par recours au salariat pour les structures uni- ou bipersonnelles (cases grisées ; exemple de lecture : en moyenne, chaque année de la période étudiée, $4 \%$ des exploitants passent d'une structure de type 1_0 à une structure de type 1_S l'année suivante), soit à un agrandissement par le passage d'une structure bipersonnelle (types 2_) à pluripersonnelle (type 3), ceci concerne notamment des exploitants dans des structures bipersonnelles ayant un apport de travail familial (2_F et 2_M) qui dans $3,6 \%$ des cas deviennent pluripersonnelles du fait de l'installation d'un aide familial ; tous les autres cas sont inférieurs à $1 \%$. Les cases à gauche de la diagonale correspondent à une régression, les fréquences de changement significatives (cases grisées) correspondent soit à la disparition du salariat (par exemple environ $10 \%$ des exploitants de type 1_S et 1_M voient la structure de l'exploitation régresser en 1_0), soit à une diminution de la main-d'œuvre familiale.

Les entrées se font majoritairement dans le type le plus simple (1_0, un seul exploitant sans conjoint ou aide familial ou salarié actif), mais la présence de salariés dès l'entrée (1_S) est un cas significatif pour les entrées dans une exploitation unipersonnelle ( $8 \%$ des entrées totales). Près d'1/3 des entrées se font directement dans 
Tableau 9. Matrice des transitions entre types de structure d'exploitation (2003-2013)

\begin{tabular}{|c|c|c|c|c|c|c|c|c|c|c|c|c|}
\hline $\mathrm{N}+1$ & $1 \_0$ & 1_F & 1_S & 1_M & 2_0_A & 2_0_B & 2_F & $2 \_S$ & $2 \_M$ & 3 & $\begin{array}{c}\text { Sorties } \\
(e n \mathrm{~N}+1)\end{array}$ & $\begin{array}{l}\text { Effectif } \\
\text { moyen }\end{array}$ \\
\hline $1 \_0$ & 88,5 & 0,7 & 4,0 & 0,1 & 0,4 & 0,2 & 0,1 & 0,2 & 0,0 & 0,2 & 5,8 & 223974 \\
\hline 1_F & 4,8 & 81,8 & 0,4 & 3,8 & 0,9 & 0,3 & 0,6 & 0,2 & 0,1 & 0,4 & 6,8 & 42156 \\
\hline $1 \_S$ & 9,7 & 0,1 & 83,9 & 0,7 & 0,2 & 0,1 & 0,0 & 1,1 & 0,0 & 0,2 & 4,0 & 77619 \\
\hline 1_M & 0,9 & 10,4 & 4,5 & 76,9 & 0,4 & 0,1 & 0,3 & 0,6 & 0,4 & 0,4 & 5,1 & 14748 \\
\hline $\begin{array}{l}\text { 1_(en } \\
\text { effectifs) }\end{array}$ & 9685 & 3179 & 9791 & 2369 & 1490 & 667 & 521 & 1475 & 101 & 831 & 19714 & 358497 \\
\hline 2_0_A & 2,0 & 0,1 & 0,3 & 0,0 & 86,0 & 0,1 & 0,3 & 7,1 & 0,0 & 1,4 & 2,6 & 49249 \\
\hline 2_0_B & 5,5 & 0,1 & 0,6 & 0,0 & 0,7 & 77,9 & 0,7 & 6,6 & 0,1 & 1,7 & 6,2 & 14490 \\
\hline 2 F & 1,6 & 1,0 & 0,2 & 0,2 & 2,1 & 4,5 & 76,5 & 0,7 & 4,7 & 3,6 & 5,0 & 8933 \\
\hline 2_S & 1,1 & 0,0 & 3,0 & 0,1 & 6,9 & 1,9 & 0,1 & 80,8 & 0,3 & 2,2 & 3,7 & 39678 \\
\hline 2_M & 0,7 & 0,7 & 1,6 & 1,1 & 0,4 & 0,6 & 9,4 & 5,8 & 71,3 & 3,6 & 4,9 & 3724 \\
\hline 2_effectif & 2387 & 179 & 1502 & 99 & 45396 & 12515 & 7473 & 36791 & 3209 & 2264 & 4276 & 116092 \\
\hline 3 & 0,7 & 0,1 & 0,3 & 0,0 & 1,7 & 1,0 & 0,2 & 2,1 & 0,1 & 89,7 & 4,2 & 54711 \\
\hline $\begin{array}{l}\text { Entrées } \\
\text { (année N) }\end{array}$ & 56,8 & 3,1 & 8,0 & 0,4 & 8,2 & 4,3 & 1,6 & 6,1 & 0,5 & 10,9 & 0,0 & 19412 \\
\hline
\end{tabular}

Note : en $\%$ des lignes.

Source : MSA, COTNS, traitement particulier, US-ODR.

des exploitations familiales existantes des types 2_ (soit 4018 personnes sur 19412 entrants annuellement en moyenne sur la période) et 3 _ (2 116 personnes). Soit ces exploitations existent déjà sous une forme pluripersonnelle (généralement sociétaire) avant l'entrée d'un nouvel exploitant (c'est le cas des $10,9 \%$ des entrées qui se font dans le cas 3), soit parallèlement à l'installation on observe un passage des cas $1_{-}$ vers les cas 2_ ou des cas 2_ vers le cas 3_. À ces entrées correspondent donc logiquement des passages d'une forme unipersonnelle à bipersonnelle (4 253 cas, en regroupant les divers type 1_ et de même les types 2_) ou à une forme de type 3 (831, lorsqu'avec l'entrée d'un enfant est créée une structure sociétaire où est associée également la conjointe de l'exploitant) et des passages du type 2 à 3 (2 264 personnes). En résumé, nous avons par année 6134 entrées dans des structures complexes et en regard 7348 transitions de personnes déjà exploitantes vers des structures complexes (logiquement ce chiffre est plus élevé que celui des entrées car certaines exploitations bipersonnelles résultent de fusion d'exploitations existantes).

Les passages d'une exploitation unipersonnelle (cas 1_) vers une pluripersonnelle (exploitations associant plusieurs exploitants : cas 2_ et 3_) sont rares, un peu moins lorsqu'il existe un support familial (les types 1_F et 1_M devenant, suite à une installation, des types 2 _ voire 3 _dans respectivement 2,5 et $2,2 \%$ des cas en moyenne par an) ou salarié (1\% des 1_S deviennent des 2_S, chaque année). Les passages de 2 exploitants vers 3 ou plus ne se font que dans des exploitations ayant un complément (support) de main-d'œuvre, qu'il soit familial, salarial ou mixte (il s'agit alors soit de l'intégration comme exploitant d'un aide familial ou d'un salarié, soit du fait que la croissance du nombre d'exploitants suppose une taille économique minimale impliquant du travail salarié, au moins de façon saisonnière). La régression du type 2_ vers 1_ ne se fait qu'entre cas sans support de main-d'œuvre 
(2_0 vers 1_0). Il ressort de l'analyse de ces différents mouvements une polarité entre des exploitations unipersonnelles et des exploitations familiales complexes.

Les changements fréquents concernent notamment, nous l'avons vu, les passages entre type d'exploitation sans support salarié vers le support salarié. Par contre, nous constatons très peu de transitions du support salarial vers un support mixte. Ces dynamiques sont cohérentes avec la substitution du travail familial par du travail salarié que montre, par ailleurs, la comparaison des recensements agricoles de 2000 et 2010 .

Il faut regarder les effectifs pour avoir une vue plus concrète des changements. Ainsi, l'effectif annuel moyen pour la période étudiée correspondant au type 1_0 (exploitation pluripersonnelle sans support) est de 223974 personnes. Au bout d'un an, le type d'exploitation n'a pas changé pour 198150 chefs (état stable, $88,5 \%) ; 12946(5,9 \%)$ ont changé de type ; 12878 (5,8\%) sont sortis du champ de la population étudiée et ont quitté une carrière agricole ; tandis que 12430 proviennent d'un autre type, il s'agit de régressions, soit par l'abandon du salariat (7 490 cas venant du type 1_S) soit par la disparition d'une exploitation bipersonnelle sans support ( 1798 personnes venant des types 2_0_A et 2_0_B), et 11029 nouveaux chefs sont entrés dans le champ de la population étudiée. Si l'on considère, l'ensemble des cas de changement avec entrée de salariés, il s'agit de 13552 personnes (dont 8959 passages du type 1_0 au type 1_S), tandis que le cas inverse ne concerne que 5431 personnes. Si l'on considère globalement le type unipersonnel, en cumulant les 4 premières lignes du tableau précédent, qui concernent en moyenne 2/3 des exploitants, $86 \%$ de ceux-ci restent dans ce type. Si l'on songe qu'il s'agit de taux annuels, un taux de sortie de 5,5\% représente un rythme important, comme les changements internes au type $(7 \%)$; les passages vers un type avec plusieurs exploitants associés ne sont pas négligeables avec un taux annuel de 1,5\%.

À l'exception de l'âge des départs en retraite, les taux de stabilité par types varient peu par classes d'âge. Ainsi les taux de stabilité pour les 40 ans et moins (âge en $\mathrm{N}+1$ ) sont proches des taux pour

\section{Tableau 10. Taux de stabilité et de sortie selon les types de structure d'exploitation et l'âge}

\begin{tabular}{lcccccc}
\hline $\begin{array}{l}\text { Type de } \\
\text { structure }\end{array}$ & $\begin{array}{c}\text { Taux } \\
\text { stabilité }\end{array}$ & $\begin{array}{c}\text { Taux de sortie } \\
\text { du champ }\end{array}$ & $\begin{array}{c}\text { Taux de sortie } \\
\text { du type }\end{array}$ & $\begin{array}{c}\text { Taux de } \\
\text { stabilité, } 40 \text { ans } \\
\text { et moins }\end{array}$ & $\begin{array}{c}\text { Taux de sortie } \\
\text { du champ, } \\
\text { 40 ans et moins }\end{array}$ & $\begin{array}{c}\text { Taux de sortie } \\
\text { du champ, } \\
\text { 61 ans et plus }\end{array}$ \\
\hline 1_0 & 88,5 & 5,8 & 5,8 & 87,6 & 3,3 & 18,1 \\
1_F & 81,8 & 6,8 & 11,4 & 80,3 & 2,3 & 36,8 \\
1_M & 76,9 & 5,1 & 18,0 & 74,3 & 2,0 & 28,0 \\
1_S & 83,9 & 4,0 & 12,2 & 83,5 & 2,5 & 14,8 \\
2_0_A & 86,0 & 2,6 & 11,4 & 86,4 & 1,8 & 26,5 \\
2_0_B & 77,9 & 6,2 & 15,9 & 78,7 & 0,9 & 30,2 \\
2_F & 76,5 & 5,0 & 18,5 & 75,8 & 0,7 & 42,9 \\
2_M & 71,3 & 4,9 & 23,9 & 67,6 & 1,0 & 30,8 \\
2_S & 80,8 & 3,7 & 15,5 & 79,8 & 1,5 & 24,4 \\
3 & 89,7 & 4,2 & 6,1 & 90,5 & 1,3 & 32,8 \\
Ensemble & 85,7 & 4,9 & 9,4 & 85,4 & 2,4 & 21,0 \\
\hline
\end{tabular}

Note : en $\%$. 
l'ensemble. Les taux de sortie des 40 ans et moins correspondent à des changements en milieu de carrière, au total plus de $2 \%$ par an; ce taux est plus élevé lorsque l'exploitant est seul (ce qui est aussi et de loin la forme d'entrée la plus fréquente). Après 60 ans, le taux de sortie annuel est de $21 \%$ et il est le plus élevé lorsqu'il y a un conjoint collaborateur ou un aide familial qui peut prendre la succession (1_F et 2_F) (tableau 10).

\section{1. Étude des états stables}

Les états stables (pas de changement du type de structure ou collectif de travail d'une année sur l'autre) sont décrits avec les critères suivants : l'âge médian des individus concernés, le statut de célibataire ou non, la superficie agricole détenue à titre individuel et l'évolution de celle-ci. Pour tous les types, la stabilité n'exclut pas un accroissement annuel de superficie de 1 à $3 \%$. Ce dernier score

Tableau 11. Stabilité des structures d'exploitation entre $\mathrm{N}$ et $\mathrm{N}+1$, Hommes

\begin{tabular}{lcccccc}
\hline $\begin{array}{l}\text { Type de } \\
\text { structure }\end{array}$ & Chefs H & $\%$ total Chefs & $\begin{array}{c}\text { Dont } \\
\text { célibataires }\end{array}$ & Âge médian & $\begin{array}{c}\text { Superficie } \\
\text { moyenne (ha) }\end{array}$ & $\begin{array}{c}\text { Évolution } \\
\text { superficie } \\
\text { moyenne (par an) }\end{array}$ \\
\hline 1_0 & 149992 & $76 \%$ & $53 \%$ & 48 & 44 & $2 \%$ \\
1_F & 29969 & $87 \%$ & $5 \%$ & 53 & 61 & $1 \%$ \\
1_M & 10368 & $92 \%$ & $2 \%$ & 52 & 59 & $1 \%$ \\
1_S & 53096 & $82 \%$ & $35 \%$ & 47 & 50 & $2 \%$ \\
2_0_A & 28618 & $68 \%$ & $35 \%$ & 46 & 53 & $1 \%$ \\
2_0_B & 7649 & $68 \%$ & $58 \%$ & 36 & 57 & $3 \%$ \\
2_F & 6185 & $91 \%$ & $33 \%$ & 48 & 63 & $1 \%$ \\
2_M & 2412 & $91 \%$ & $24 \%$ & 50 & 69 & $1 \%$ \\
2_S & 21636 & $67 \%$ & $31 \%$ & 46 & 55 & $2 \%$ \\
3 & 34537 & $70 \%$ & $39 \%$ & 44 & 53 & $3 \%$ \\
\hline
\end{tabular}

Source : MSA, COTNS, traitement particulier, US-ODR.

Tableau 12. Stabilité des structures d'exploitation entre $\mathrm{N}$ et $\mathrm{N}+1$, Femmes

\begin{tabular}{lrrcccc}
\hline $\begin{array}{l}\text { Type de } \\
\text { structure }\end{array}$ & Chefs F & $\%$ total Chefs & $\begin{array}{c}\text { Dont } \\
\text { célibataires }\end{array}$ & Âge médian & $\begin{array}{c}\text { Superficie } \\
\text { moyenne (ha) }\end{array}$ & $\begin{array}{c}\text { Évolution } \\
\text { superficie } \\
\text { moyenne (par an) }\end{array}$ \\
\hline 1_0 & 48145 & $24 \%$ & $18 \%$ & 56 & 29 & $-1 \%$ \\
1_F & 4529 & $13 \%$ & $6 \%$ & 51 & 40 & $1 \%$ \\
1_M & 949 & $8 \%$ & $4 \%$ & 49 & 32 & $1 \%$ \\
1_S & 12009 & $18 \%$ & $17 \%$ & 54 & 37 & $1 \%$ \\
2_0_A & 13734 & $32 \%$ & $12 \%$ & 45 & 39 & $2 \%$ \\
2_0_B & 3637 & $32 \%$ & $7 \%$ & 58 & 49 & $1 \%$ \\
2_F & 634 & $9 \%$ & $18 \%$ & 53 & 50 & $2 \%$ \\
2_M & 228 & $9 \%$ & $19 \%$ & 55 & 54 & $1 \%$ \\
2_S & 10440 & $33 \%$ & $10 \%$ & 48 & 44 & $2 \%$ \\
3 & 14507 & $30 \%$ & $10 \%$ & 51 & 41 & $2 \%$ \\
\hline
\end{tabular}

Source : MSA, COTNS, traitement particulier, US-ODR. 
n'est atteint que par les exploitations avec trois exploitants au moins (type 3 ) et par les bipersonnelles associant 2 générations (2_0_B).

Le type 1_0, le plus stable avec le type 3_ (bien moins fréquent), paraît jouer un rôle différent dans les carrières masculines et féminines. Dans le premier cas, la proportion de célibataires et l'âge médian suggèrent qu'il s'y retrouve tant des jeunes en début de parcours que des exploitants vers la fin de leur carrière (50\% ont plus de 48 ans). Tandis que dans le cas des femmes, il s'agit plutôt de situations de personnes âgées (50\% ont plus de 56 ans) et bien moins sont célibataires. Dans ce cas, l'évolution de la superficie moyenne détenue est négative (tableaux 11 et 12).

Les femmes sont rarement présentes en tant que chef d'exploitation (la population étudiée, rappelons-le, est celle des chefs d'exploitation ou exploitants nonsalariés) dans les exploitations avec support familial. De fait, comme on le sait, les « conjoints participants aux travaux » sont essentiellement du genre féminin et dans ce cas les chefs d'exploitation du genre masculin.

\section{Entrées selon les types de structure d'exploitation}

En considérant les groupes où dominent les célibataires et où l'âge médian est inférieur à 40 ans, nous pouvons dire que l'entrée des jeunes exploitants se fait de préférence dans des exploitations unipersonnelles sans support (type 1_0) ou dans des exploitations pluripersonnelles. L'entrée dans le type le plus simple 1_0 est surtout le fait des jeunes hommes: $50 \%$ des entrants dans ce type ont 34 ans au plus (détenant en moyenne 19 ha) tandis que $50 \%$ des femmes entrant dans ce type le font à partir de 50 ans (détenant en moyenne $28 \mathrm{ha}$ ), prenant alors en général la succession du conjoint (tableau 13). Une majorité de femmes entre dans l'agriculture avant 40 ans seulement dans le cas des exploitations les plus complexes (2_F, $\mathrm{M}$ ou $\mathrm{S}$ et 3 ) ou en s'installant avec quelqu'un de la même génération (2_0_A). Une part importante des entrants sont salariés agricoles l'année précédente leur installation comme exploitant : $29 \%$ des entrants en 2003 et $34 \%$ en 2013 ont eu, selon la base SISAL, un contrat de travail l'année précédente (tableau 14). Une partie de ces entrants ont d'abord été salariés (au lieu d'aide familial) sur l'exploitation

Tableau 13. Caractéristiques moyennes des entrées pour une année et par genre

\begin{tabular}{lrccc|cccc}
\hline \multirow{2}{*}{$\begin{array}{l}\text { Type de } \\
\text { structure }\end{array}$} & \multicolumn{4}{c|}{ Hommes } & \multicolumn{4}{c}{ Femmes } \\
\cline { 2 - 8 } & Chefs & $\begin{array}{c}\text { Dont } \\
\text { célibataires }\end{array}$ & $\begin{array}{c}\text { Âge } \\
\text { médian }\end{array}$ & $\begin{array}{c}\text { Superficie } \\
\text { moyenne }\end{array}$ & Chefs & $\begin{array}{c}\text { Dont } \\
\text { célibataires }\end{array}$ & $\begin{array}{c}\text { Áge } \\
\text { médian }\end{array}$ & $\begin{array}{c}\text { Superficie } \\
\text { moyenne }\end{array}$ \\
\hline 1_0 & 7251 & $69 \%$ & 34 & 19 & 3829 & $25 \%$ & 50 & 28 \\
1_F & 305 & $31 \%$ & 40 & 32 & 307 & $10 \%$ & 50 & 39 \\
1_M & 47 & $15 \%$ & 44 & 36 & 27 & $11 \%$ & 48 & 39 \\
1_S & 994 & $58 \%$ & 37 & 23 & 556 & $21 \%$ & 50 & 30 \\
2_0_A & 530 & $87 \%$ & 26 & 39 & 293 & $19 \%$ & 53 & 43 \\
2_0_B & 686 & $66 \%$ & 33 & 25 & 894 & $21 \%$ & 40 & 31 \\
2_F, M, S & 988 & $78 \%$ & 27 & 39 & 602 & $22 \%$ & 42 & 35 \\
3 & 1342 & $84 \%$ & 26 & 41 & 762 & $25 \%$ & 41 & 34 \\
\hline
\end{tabular}

Source : MSA, COTNS, traitement particulier, US-ODR. 
Tableau 14. Entrées comme exploitants de salariés agricoles en 2003 et 2013

\begin{tabular}{lcrr|rrc}
\hline Type de structure & Entrants 2003 & dont SISAL 2002 & $\%$ & Entrants 2013 & dont SISAL 2012 & $\%$ \\
\hline 1_0 & 11215 & 2992 & $27 \%$ & 6473 & 1852 & $29 \%$ \\
1_F & 849 & 93 & $11 \%$ & 351 & 66 & $19 \%$ \\
1_S & 1685 & 517 & $31 \%$ & 1109 & 432 & $39 \%$ \\
1_M & 108 & 20 & $19 \%$ & 40 & 10 & $25 \%$ \\
2_0_A & 1451 & 300 & $21 \%$ & 1377 & 283 & $21 \%$ \\
2_0_B & 684 & 208 & $30 \%$ & 679 & 261 & $38 \%$ \\
2_F/S/M et 3 & 4098 & 1692 & $41 \%$ & 2973 & 1560 & $52 \%$ \\
Total & 20090 & 5822 & $29 \%$ & 13002 & 4464 & $34 \%$ \\
\hline
\end{tabular}

Source : MSA, COTNS, traitement particulier, US-ODR.

Tableau 15. Caractéristiques moyennes annuelles des départs, toute population et par sexe

\begin{tabular}{lrcc|rcc|rrrr}
\hline $\begin{array}{l}\text { Type de } \\
\text { structure }\end{array}$ & \multicolumn{3}{c|}{ Toute population } & \multicolumn{3}{|c|}{ Hommes } & \multicolumn{4}{c}{ Femmes } \\
\cline { 2 - 11 } & Chefs & $\begin{array}{c}\text { Áge } \\
\text { médian }\end{array}$ & $\begin{array}{c}\text { Superficie } \\
\text { moyenne }\end{array}$ & Chefs & $\begin{array}{c}\text { Céliba- } \\
\text { taires }\end{array}$ & $\begin{array}{c}\text { Áge } \\
\text { médian }\end{array}$ & Chefs & $\begin{array}{c}\text { Céliba- } \\
\text { taires }\end{array}$ & Veuves & $\begin{array}{c}\text { Áge } \\
\text { médian }\end{array}$ \\
\hline 1_0 & 12922 & 60 & 23 & 8148 & $41 \%$ & 58 & 4774 & $11 \%$ & $11 \%$ & 61 \\
1_F & 2936 & 60 & 48 & 2586 & $2 \%$ & 60 & 350 & $4 \%$ & $11 \%$ & 59 \\
1_M & 771 & 60 & 46 & 704 & $1 \%$ & 60 & 67 & $3 \%$ & $12 \%$ & 58 \\
1_S & 3073 & 59 & 29 & 2103 & $27 \%$ & 57 & 970 & $10 \%$ & $13 \%$ & 61 \\
2_0_A & 1287 & 53 & 35 & 819 & $32 \%$ & 56 & 468 & $15 \%$ & $2 \%$ & 49 \\
2_0_B & 907 & 61 & 47 & 333 & $21 \%$ & 61 & 574 & $2 \%$ & $11 \%$ & 61 \\
2_F_M & 634 & 60 & 52 & 539 & $8 \%$ & 60 & 95 & $6 \%$ & $9 \%$ & 61 \\
2_S & 1471 & 59 & 40 & 815 & $19 \%$ & 59 & 656 & $6 \%$ & $8 \%$ & 60 \\
3 & 2301 & 60 & 40 & 1400 & $17 \%$ & 60 & 901 & $6 \%$ & $7 \%$ & 61 \\
\hline
\end{tabular}

Source : MSA, COTNS, traitement particulier, US-ODR.

agricole dans laquelle ils entrent, les entrées de salariés étant les plus fréquentes pour les types 2_F/S/M et 3.

\section{Sorties selon les types de structure d'exploitation}

Tant pour les hommes que pour les femmes, l'âge médian de sortie se situe autour de 60 ans (tableau 15). Il est le plus bas pour les hommes dans les cas 1_0, 1_S et 2_A, à partir desquels se font des reconversions professionnelles. Pour les femmes, dans le cas 2_A, $50 \%$ des sorties se font avant 49 ans, ce qui montre une certaine instabilité de ces associations (une partie de ces femmes prenant sans doute un travail salarié une fois les enfants scolarisés).
Un nombre important d'hommes sortent célibataires (jusqu'à $41 \%$ pour le cas 1_0). Ainsi semble persister un phénomène social caractéristique de la paysannerie de la seconde moitié du $\mathrm{XX}^{\mathrm{e}}$ siècle. Le célibat est nettement moins marqué pour les femmes (dont beaucoup sont entrées en agriculture par le mariage). Tandis que moins de $2 \%$ des hommes sortant sont veufs, les femmes sortantes sont nettement plus souvent veuves.

Les taux de sortie annuels par type et par âge jusqu'à 55 ans (sorties précoces) sont constants et varient de $3 \%$ pour les types 1_à 1 ou $2 \%$ pour les autres. Entre 56 et 60 ans, ils s'établissent entre 6 et $9 \%$ /an. À partir de 61 ans, les taux de 
sortie moyens annuels augmentent fortement, mais varient aussi fortement selon les types de structures : de seulement $14 \%$ et $18 \%$ pour 1_S et 1_0 (une partie des exploitations 1_0 sont des exploitations de subsistance) à $43 \%$ pour le type 2_F ; les autres cas se situant entre $25 \%$ et $35 \%$ (types 1_F et 3 ). Ainsi les exploitations où cohabitent 2 générations (types 2_F et 3) permettent des prises de retraite plus jeune (ce qui ne signifie pas toujours un arrêt du travail sur l'exploitation).

\section{Caractéristiques des individus dans les changements de structure d'exploitation les plus fréquents}

Après les transitions sans changement, les entrées et les sorties, nous étudions les transitions avec changement de structure d'exploitation les plus fréquentes (au moins $5 \%$ de la population d'un type), en distinguant les transitions avec gain de force de travail (progression), en grisé dans les tableaux suivants, et celles où il y a perte de force de travail (régression),

Tableau 16. Caractéristiques des changements d'état les plus fréquents (hommes)

\begin{tabular}{ccrrccc}
\hline $\begin{array}{c}\text { Type de struc- } \\
\text { ture année N }\end{array}$ & $\begin{array}{c}\text { Type de structure } \\
\text { année N +1 }\end{array}$ & Chefs & $\begin{array}{c}\text { Céliba- } \\
\text { taires }\end{array}$ & $\begin{array}{c}\text { Âge } \\
\text { médian }\end{array}$ & $\begin{array}{c}\text { Superficie } \\
\text { moyenne }\end{array}$ & $\begin{array}{c}\text { Évolution moyenne de la } \\
\text { superficie entre les deux années }\end{array}$ \\
\hline 1_S & 1_0 & 6110 & $41 \%$ & 46 & 51 & $0 \%$ \\
1_M & 1_F & 1377 & $3 \%$ & 52 & 65 & $0 \%$ \\
2_0_A & 2_S & 2274 & $29 \%$ & 45 & 57 & $2 \%$ \\
2_0_B & 1_0 & 700 & $62 \%$ & 37 & 54 & $82 \%$ \\
2_0_B & 2_S & 658 & $53 \%$ & 36 & 59 & $6 \%$ \\
2_S & 2_0_A & 1788 & $28 \%$ & 45 & 59 & $1 \%$ \\
2_M & 2_F & 318 & $29 \%$ & 48 & 72 & $2 \%$ \\
2_M & 2_S & 186 & $35 \%$ & 41 & 60 & $7 \%$ \\
\hline
\end{tabular}

Notes : changements représentant en moyenne plus de $5 \%$ de la population de l'année $\mathrm{N}$; lignes classées dans l'ordre de la fréquence du changement.

Source : MSA, COTNS, traitement particulier, US-ODR.

Tableau 17. Caractéristiques des changements d'état les plus fréquents (femmes)

\begin{tabular}{ccrrccc}
\hline $\begin{array}{c}\text { Type de struc- } \\
\text { ture année N }\end{array}$ & $\begin{array}{c}\text { Type de struc- } \\
\text { ture année N +1 }\end{array}$ & Chefs & $\begin{array}{c}\text { Céliba- } \\
\text { taires }\end{array}$ & $\begin{array}{c}\text { Âge } \\
\text { médian }\end{array}$ & $\begin{array}{c}\text { Superficie } \\
\text { moyenne }\end{array}$ & $\begin{array}{c}\text { Évolution moyenne de la } \\
\text { superficie entre les deux années }\end{array}$ \\
\hline 1_F & 1_0 & 426 & $5 \%$ & 56 & 39 & $-11 \%$ \\
1_S & 1_0 & 1377 & $19 \%$ & 54 & 34 & $-2 \%$ \\
1_M & 1_F & 168 & $6 \%$ & 49 & 38 & $2 \%$ \\
1_M & 1_S & 88 & $4 \%$ & 54 & 37 & $-8 \%$ \\
2_0_A & 2_S & 1212 & $11 \%$ & 45 & 43 & $2 \%$ \\
2_0_B & 2_S & 294 & $9 \%$ & 58 & 52 & $3 \%$ \\
2_F & 2_0_B & 81 & $9 \%$ & 56 & 48 & $4 \%$ \\
2_S & 2_0_A & 935 & $10 \%$ & 45 & 45 & $2 \%$ \\
2_M & 2_F & 34 & $18 \%$ & 52 & 54 & $5 \%$ \\
2_M & 2_S & 31 & $14 \%$ & 54 & 53 & $11 \%$ \\
\hline
\end{tabular}

Notes : changements représentant en moyenne plus de $5 \%$ de la population de l'année $\mathrm{N}$; lignes classées dans l'ordre de la fréquence du changement. 
laissées en blanc. Le cas du passage 2_M à 2_S paraît aussi un cas de croissance, du moins au regard de la superficie détenue (+7\% en moyenne).

Les tableaux 16 et 17 concernent les changements les plus fréquents entre une année $\mathrm{N}$ et $\mathrm{N}+1$, par genre. Le cas le plus nombreux, tant pour les hommes que pour les femmes, est le passage 1_S à 1_0 (suppression du support salarié) qui correspond à une régression précédent une sortie et qui en particulier pour les hommes peut s'effectuer assez jeune (dans $50 \%$ des cas avant ou à 46 ans), en particulier pour les célibataires.

Un cas remarquable est le passage 2_0_B à 1_0 (régression) qui correspond principalement au départ d'un exploitant à la retraite dans une association parentenfant ; celui qui reste, presque toujours un homme ayant dans $50 \%$ des cas 37 ans au plus, récupère les terres du parent qui s'en va, d'où une évolution très importante de la superficie détenue par l'exploitant ( $t a-$ bleau 16). Deux autres cas remarquables sont les passages 2_0_B à 2_S (ajout d'un support salarié) et 2_M à 2_S qui s'accompagnent d'une hausse significative de la superficie. Les indicateurs sur l'état matrimonial et l'âge médian permettent de décrire ces situations : dans le premier cas, il s'agit majoritairement, dans le cas des hommes, de jeunes exploitants et de célibataires et l'inverse pour les femmes (ces situations étant généralement des associations mère-fils).

\section{Trajectoires de la cohorte des entrants en 2003}

Nous considérons maintenant la cohorte des entrées en 2003 (20 090 individus), il s'agit du début d'une carrière comme exploitant agricole, qui peut intervenir à tout âge, que nous observons sur 10 ans, une sortie de l'activité agricole pouvant intervenir avant (36\% des cas).

L'âge médian des entrants en 2003 (tableau 18) étant globalement de 36 ans, il s'agit très majoritairement d'agriculteurs de 40 ans et moins, c'est-à-dire de «jeunes agriculteurs » pouvant bénéficier, sous conditions, d'une exonération de cotisation AMEXA et d'une aide à l'installation (Dotation jeune agriculteur [DJA]). Toutefois une partie appréciable

Tableau 18. Statistiques individuelles des entrants en 2003, par type de structure

\begin{tabular}{lrllcccccc}
\hline $\begin{array}{l}\text { Type } \\
\text { de } \\
\text { struc- } \\
\text { ture }\end{array}$ & $\begin{array}{r}\text { Ex- } \\
\text { ploi- } \\
\text { tants }\end{array}$ & $\begin{array}{c}\text { Dont } \\
\text { femmes }\end{array}$ & $\begin{array}{c}\text { Âge } \\
\text { médian }\end{array}$ & $\begin{array}{c}\text { Superficie } \\
\text { moyenne } \\
\text { (ha) }\end{array}$ & $\begin{array}{c}\text { Céliba- } \\
\text { taires }\end{array}$ & $\begin{array}{c}\text { Dont } \\
\text { cotisants } \\
\text { solidaires }\end{array}$ & $\begin{array}{c}\text { Dont } \\
\text { exploitants } \\
\text { à titre } \\
\text { secondaire }\end{array}$ & $\begin{array}{c}\text { Dont } \\
\text { transferts } \\
\text { entre } \\
\text { époux }\end{array}$ & $\begin{array}{c}\% \text { des } 40 \text { ans } \\
\text { et moins, exo- } \\
\text { nérés jeunes } \\
\text { agriculteurs }\end{array}$ \\
\hline 1_0 & 11215 & $35 \%$ & 37 & 23,4 & $47 \%$ & $11 \%$ & $32 \%$ & $17 \%$ & $58 \%$ \\
1_F & 849 & $56 \%$ & 51 & 37,85 & $6 \%$ & $0 \%$ & $6 \%$ & $44 \%$ & $84 \%$ \\
1_S & 1685 & $34 \%$ & 40 & 25,71 & $36 \%$ & $1 \%$ & $23 \%$ & $12 \%$ & $72 \%$ \\
1_M & 108 & $30 \%$ & 47 & 39,08 & $2 \%$ & $0 \%$ & $5 \%$ & $19 \%$ & $77 \%$ \\
2_0_A & 1451 & $62 \%$ & 36 & 30,89 & $24 \%$ & $0 \%$ & $10 \%$ & $2 \%$ & $85 \%$ \\
2_0_B & 684 & $39 \%$ & 28 & 39,22 & $53 \%$ & $0 \%$ & $9 \%$ & $19 \%$ & $88 \%$ \\
2_F & 519 & $25 \%$ & 26 & 41,97 & $64 \%$ & $0 \%$ & $3 \%$ & $7 \%$ & $92 \%$ \\
2_S & 1075 & $44 \%$ & 33 & 34,61 & $40 \%$ & $0 \%$ & $9 \%$ & $6 \%$ & $87 \%$ \\
2_M & 152 & $20 \%$ & 27 & 44,23 & $63 \%$ & $0 \%$ & $3 \%$ & $7 \%$ & $94 \%$ \\
3 & 2352 & $46 \%$ & 31 & 36 & $43 \%$ & $0 \%$ & $4 \%$ & $4 \%$ & $92 \%$ \\
Total & 20090 & $39 \%$ & 36 & 28,08 & $42 \%$ & $6 \%$ & $22 \%$ & $14 \%$ & $71 \%$ \\
\hline
\end{tabular}


des installations sont tardives, comme nous l'avons vu. Une partie significative de ces installations tardives correspond à des transferts entre époux au moment de la retraite de celui qui est exploitant, le plus souvent au bénéfice des femmes. Au total, les transferts entre époux représentent $14 \%$ des installations et le tiers des installations d'actifs de plus de 40 ans ( $c f$. Allaire et Maigné, 2013). Ces transferts concernent surtout les exploitations unipersonnelles (l'épouse bénéficiant du transfert n'étant pas déjà exploitant) et s'observent plus particulièrement lorsqu'un aide familial est présent (type 1_F et 1_M). C'est là l'un des deux types de structures caractéristiques des entrées féminines avec, pour les plus jeunes, l'association avec un conjoint déjà agriculteur (type 2_A).

La matrice des changements d'état (tableau 19) montre des phénomènes comparables à ceux que nous avons précédemment mis en évidence, avec un taux légèrement moins important d'états stables. On remarque cependant que pour certains types, le taux de sortie est supérieur au taux de l'ensemble de la population ( $c f$. la dernière colonne reprise du tableau 9). Ceci concerne les types d'exploitations (1_0 et
1_S), pour lesquels une partie significative des entrées sont des entrées tardives de femmes qui cessent leur activité au bout de quelques années.

L'intensité des transitions est mise en évidence par un dégradé de gris. Durant la décennie observée, la moitié des exploitants entrés en 2003 n'a pas connu de changement de structure et un quart n'a connu qu'un seul changement de type de structure.

\section{Trajectoires de la cohorte des sortants en 2012}

Il faut d'abord noter l'importance des sorties précoces, déjà vue. À peine la majorité de ces sortants a au moins 60 ans $(53 \%)$ (tableau 20).

Les sorties de célibataires sont importantes dans les cas sans support, soit en exploitation unipersonnelle (1_0) (50\% du total des sorties), soit pour une association entre 2 personnes de la même génération (2_0_A). À l'inverse, les célibataires sont rares dans les sorties pour les cas 2_0_B, 2_F, 2_M qui associent un jeune (comme exploitant pour 2_0_B ou aide familial dans les autres types) avec un ou les deux

Tableau 19. Matrice de transition de la cohorte des entrants en 2003, période 2003-2013

\begin{tabular}{|c|c|c|c|c|c|c|c|c|c|c|c|c|c|}
\hline$N^{N+1}$ & $\begin{array}{l}\text { Effectif } \\
\text { moyen }\end{array}$ & $1 \_0$ & 1_F & 1_S & 1_M & 2_0_A & 2_O_B & 2_F & $2 \_s$ & 2_M & 3 & $\begin{array}{c}\text { Taux } \\
\text { Sor- } \\
\text { tie } \\
\end{array}$ & $\begin{array}{c}\text { Taux Sor- } \\
\text { tie Toute } \\
\text { population }\end{array}$ \\
\hline $1 \_0$ & 7596 & 86,7 & 0,7 & 4,7 & 0,1 & 0,5 & 0,3 & 0,1 & 0,2 & 0,0 & 0,4 & 6,4 & 5,8 \\
\hline 1_F & 585 & 7,9 & 79,2 & 0,7 & 4,5 & 0,9 & 0,3 & 0,2 & 0,2 & 0,0 & 0,3 & 5,8 & 6,8 \\
\hline 1_S & 2180 & 10,3 & 0,2 & 81,7 & 0,7 & 0,3 & 0,1 & 0,0 & 0,9 & 0,0 & 0,3 & 5,6 & 4,0 \\
\hline 1_M & 160 & 1,9 & 9,8 & 7,4 & 73,1 & 0,6 & 0,3 & 0,3 & 1,2 & 0,3 & 0,3 & 4,9 & 5,1 \\
\hline 2_0_A & 1342 & 1,9 & 0,0 & 0,3 & 0,0 & 86,1 & 0,1 & 0,2 & 7,5 & 0,0 & 1,3 & 2,6 & 2,6 \\
\hline 2_0_B & 738 & 4,9 & 0,0 & 0,7 & 0,0 & 0,6 & 80,5 & 0,7 & 6,0 & 0,1 & 1,7 & 4,8 & 6,2 \\
\hline 2_F & 324 & 1,8 & 0,6 & 0,3 & 0,0 & 1,7 & 8,7 & 75,7 & 1,4 & 4,2 & 3,6 & 1,9 & 5,0 \\
\hline 2_S & 1142 & 1,1 & 0,0 & 3,7 & 0,1 & 6,3 & 2,9 & 0,1 & 79,8 & 0,5 & 1,8 & 3,7 & 3,7 \\
\hline 2_M & 104 & 0,9 & 0,4 & 2,5 & 1,0 & 0,2 & 1,4 & 11,1 & 6,6 & 68,8 & 3,1 & 3,9 & 4,9 \\
\hline 3 & 2054 & 0,6 & 0,0 & 0,2 & 0,0 & 1,7 & 1,5 & 0,2 & 2,4 & 0,1 & 90,2 & 3,1 & 4,2 \\
\hline
\end{tabular}

Note : En \% des lignes, sauf effectif. 
Tableau 20. Statistiques individuelles des sortants en 2012, par type de structure

\begin{tabular}{lrrrcccc}
\hline $\begin{array}{l}\text { Type de } \\
\text { structure }\end{array}$ & $\begin{array}{c}\text { Exploi- } \\
\text { tants }\end{array}$ & $\begin{array}{c}\text { Dont } \\
\text { femmes }\end{array}$ & $\begin{array}{c}\text { Dont 60 } \\
\text { ans et plus }\end{array}$ & $\begin{array}{c}\text { Superficie } \\
\text { moyenne (ha) }\end{array}$ & $\begin{array}{c}\text { Dont céli- } \\
\text { bataires }\end{array}$ & $\begin{array}{c}\text { Dont cotisants } \\
\text { solidaires }\end{array}$ & $\begin{array}{c}\text { Dont exploitants } \\
\text { à titre secondaire }\end{array}$ \\
\hline 1_0 & 10274 & $35 \%$ & $51 \%$ & 23.11 & $33 \%$ & $19 \%$ & $18 \%$ \\
1_F & 1393 & $18 \%$ & $62 \%$ & 48.02 & $4 \%$ & $0 \%$ & $4 \%$ \\
1_M & 405 & $13 \%$ & $60 \%$ & 51.13 & $3 \%$ & $0 \%$ & $2 \%$ \\
1_S & 2796 & $31 \%$ & $48 \%$ & 28.42 & $23 \%$ & $3 \%$ & $11 \%$ \\
2_0_A & 1197 & $36 \%$ & $31 \%$ & 35.18 & $32 \%$ & $0 \%$ & $8 \%$ \\
2_0_B & 804 & $59 \%$ & $79 \%$ & 51.16 & $11 \%$ & $0 \%$ & $5 \%$ \\
2_F & 226 & $24 \%$ & $70 \%$ & 60.5 & $11 \%$ & $0 \%$ & $1 \%$ \\
2_M & 119 & $21 \%$ & $71 \%$ & 56.89 & $8 \%$ & $0 \%$ & $3 \%$ \\
2_S & 1505 & $44 \%$ & $56 \%$ & 43.82 & $15 \%$ & $0 \%$ & $6 \%$ \\
3 & 2002 & $41 \%$ & $56 \%$ & 39.75 & $17 \%$ & $0 \%$ & $3 \%$ \\
Total & 20721 & $35 \%$ & $53 \%$ & 31.55 & $25 \%$ & $10 \%$ & $12 \%$ \\
\hline
\end{tabular}

Source : MSA, COTNS, traitement particulier, US-ODR.

parents ; dans ces sorties qui très majoritairement se font à l'âge de la retraite les femmes sont très minoritaires lorsqu'il y a un aide familial prêt à s'installer (2_F, 2_M) et majoritaires pour les associations entre 2 personnes de génération différente (2_0_B). Nous pouvons alors supposer que la sortie de l'homme d'une exploitation bipersonnelle se fait en même temps que l'installation d'un jeune pour former une exploitation du type 2_0_B avec la mère (qui continue ainsi de cotiser pour la retraite).

Sur 20721 sortants de 2012, 14545 étaient et 6176 n'étaient pas exploitants en 2003, soit $30 \%$ des sortants, qui sont donc sortis après une durée d'activité agricole d'au plus 10 ans.

Les changements d'état s'effectuant plutôt en début de carrière comme nous venons de le voir, la matrice des transitions (précédant la sortie) pour les sortants en 2012 (chiffres non publiés) montre des taux de stabilité plus élevés que pour l'ensemble de la population.

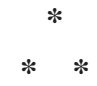

Les travaux que nous venons de présenter ont une double originalité : du point de vue de l'origine des données et de la méthode. Les données fournies par la MSA (via l'ODR) nous ont permis de suivre année après année, pendant 10 ans, une cohorte d'exploitants agricoles non salariés. La disposition d'une base longitudinale permet de caractériser pour chaque individu les changements d'état et de constituer des matrices de transition annuelles (nous présentons des matrices moyennes pour la période). Les changements étudiés concernent les exploitants et se rapportent au régime d'activité et au type de structure d'exploitation sur laquelle exerce l'exploitant considéré, différents types étant distingués selon la taille et la composition des collectifs de travail.

Le taux de pluriactivité de la population étudiée augmente à partir du milieu des années 2000. Plus du quart des entrées se font avec un statut de pluriactif. Le taux d'exploitants à titre secondaire chez les entrants est le double que pour l'ensemble de la population. C'est généralement en début de carrière que s'effectuent les transitions des catégories à titre principal et à titre secondaire vers la catégorie à titre exclusif.

La répartition des types de structures (collectifs de travail) distingués est plutôt 
stable sur la période, les exploitations unipersonnelles dominent largement. Les entrées se font très majoritairement dans le type le plus simple (un seul exploitant sans conjoint ou aide familial ou salarié actif), cependant près du tiers des entrées ont lieu dans des exploitations familiales existantes. Les taux de sortie sont les plus faibles lorsqu'il y a du travail salarié. La part des exploitations avec apport salarié augmente au détriment du support familial.

L'analyse des carrières montre comment s'effectuent, en fonction des cycles familiaux, simultanément la croissance et la régression des structures d'exploitation. Toutefois, nous avons noté l'importance des sorties précoces (environ 1/3 des sortants ont moins de 56 ans) et les entrées tardives s'agissant des carrières de femmes.

La base de données utilisée a un avantage sur les enquêtes des statistiques agricoles, celui de fournir des données annuelles exhaustives sur l'ensemble de la population agricole, que permet d'exploiter la méthodologie utilisée. La contrepartie est que l'analyse est tributaire du champ réglementaire de la population agricole et des catégories déterminées par les régimes sociaux, cadre plus instable que celui défini par les statisticiens agricoles pour les recensements et les enquêtes et qui pose des problèmes d'interprétation des évolutions. La plupart des travaux publiés sur la dynamique des structures agricoles utilisent les statistiques publiques (Agreste, Insee, Eurostat). Les conclusions qui ressortent de nos travaux peuvent renvoyer à différents travaux concernant les années 1990 ou 2000 avec des données Agreste. Par exemple, une évolution remarquable, déjà constatée à la fin des années 1990, est l'importance des sorties précoces (avant 55 ans) et des entrées tardives (après 40 ans). Du point de vue de la dynamique des structures d'exploitation, une convergence évidente des travaux récents concerne le développement du salariat agricole. Il nous a semblé cependant préférable de ne pas discuter les convergences ou les divergences d'appréciation avec les travaux basés sur des données Agreste. En effet, quoique l'on puisse rapprocher la conception statistique d'Agreste avec la conception réglementaire (MSA) de la population agricole, les champs statistiques restent très différents. Une discussion comparative aurait demandé trop de détail.

Nos résultats concernent la population agricole telle qu'elle est réglementairement constituée, ce qui n'est pas en soi sans intérêt, mais ne prend pas en compte diverses relations entre les réalités réglementaires et les structures économiques de l'activité qui peuvent relever d'associations non sociétaires entre exploitations individualisées dans cette étude ou de relations coopératives (par exemple dans le cadre de CUMA) qui constituent une forme indirecte d'apport de travail salarié non ici connue. De plus, la base de données utilisée exclut les exploitations agricoles sans exploitant non salarié, qui en 2012 constituent $20 \%$ de l'ensemble et dont les dynamiques sont mal connues. Cette définition de la population d'exploitants familiaux considérée ici étant précisée, les cas d'exploitant seul sans complément de main-d'œuvre (hors travail informel) concernent $42 \%$ de la population étudiée (en moyenne) et $57 \%$ des entrées. Ce type est le plus stable avec celui des exploitations ayant 3 exploitants ou plus ; l'évolution se faisant principalement vers une exploitation unipersonnelle avec support de travail salarié. Par ailleurs, $33 \%$ des exploitants sur l'ensemble de la période appartiennent à des exploitations familiales complexes (au moins 2 exploitants plus un support familial ou salarié), sur lesquelles s'opèrent 31,5 des entrées de nouveaux exploitants. Globalement, les dynamiques que nous avons mises en évidence concernent d'une façon différente deux 
ensembles d'exploitants et d'exploitations qui se distinguent d'un point de vue structurel par une grande majorité d'exploitation où un exploitant est seul présent (avec ou sans support familial ou salarié, 2/3 des exploitants) et une minorité d'exploitations familiales complexes. Sur le plan empirique, c'est sans doute là le résultat le plus original de notre analyse.

L'image d'une agriculture familiale dont la structuration et les mécanismes d'évolution paraissent peu différents de ceux que l'on pouvait constater dans le passé (excepté le rôle croissant du salariat) rend bien sûr imparfaitement compte des structures de production de l'agriculture actuelle; d'une part car les exploitations sous forme de société et n'employant que des salariés sont exclus du champ de l'étude; d'autre part car les structures familiales se caractérisent également par leurs relations contractuelles avec des fournisseurs de services (CUMA, groupement d'employeurs, coopératives...).

\section{RÉFÉRENCES BIBLIOGRAPHIQUES}

Allaire G., Daucé P. (1996). La préretraite en agriculture 1992-1994. Premier bilan et éléments d'impact structurel. Économie rurale, $n^{\circ} 232$, pp. 3-11.

Allaire G., Maigné E. (2013). Installation en agriculture et renouvellement de la population agricole : retour sur 10 ans de politique de développement rural. In A. Trouvé,
M. Berriet-Solliec, D. Lépicier (dir.), Le développement rural en Europe. Quel avenir pour le deuxième pilier de la Politique agricole commune? Bruxelles, P.I.E. Peter Lang, pp. 173-203.

Bernardi V., Lefebvre F. (2002). Les départs précoces de l'agriculture: mythes ou réalité ? Cahiers du CNASEA, octobre, $\mathrm{n}^{\circ} 2$. 